\title{
Cardiac BIN1 (cBIN1) is a regulator of cardiac contractile function and an emerging biomarker of heart muscle health
}

\author{
Kang Zhou ${ }^{1,2}$ \& Tingting Hong ${ }^{2,3 *}$ \\ 'Department of Cardiovascular Surgery, 2nd XiangYa Hospital, Central South University, Changsha 410011, China; \\ ${ }^{2}$ Cedars-Sinai Heart Institute, Cedars-Sinai Medical Center, Los Angeles 90048, USA, \\ ${ }^{3}$ Departments of Medicine, Cedars-Sinai Medical Center and UCLA, Los Angeles 90048, USA
}

Received August 31,2016; accepted October 6, 2016; published online November 23, 2016

\begin{abstract}
In recent decades, a cardiomyocyte membrane scaffolding protein bridging integrator 1 (BIN1) has emerged as a critical multifunctional regulator of transverse-tubule (t-tubule) function and calcium signaling in cardiomyocytes. Encoded by a single gene with 20 exons that are alternatively spliced, more than ten BIN1 protein isoforms are expressed with tissue and disease specificity. The recently discovered cardiac alternatively spliced isoform BIN1 (cBIN1 or BIN1+13+17)plays a crucial role in organizing membrane microfolds within cardiac t-tubules. These cBIN1-induced microfolds form functional dyad microdomains by trafficking L-type calcium channels (LTCC) to t-tubule membrane and recruiting ryanodine receptors (RyR) to junctional sarcoplasmic reticulum membrane. When cBIN1 is transcriptionally reduced as occurs in heart failure, cBIN1-microfolds are disrupted and fail to form LTCC and RyR couplons. As a result, impaired dyad formation limits excitation-contraction coupling thus cardiac contractility, and accumulation of orphaned leaky RyRs outside of dyads increases ventricular arrhythmias. Reduced myocardial BIN1 in heart failure is also detectable at the blood level, and plasma BIN1 level predicts heart failure progression and future arrhythmias in cardiomyopathy patients. Here we will review the recent progress in BIN1-related cardiomyocyte biology studies and discuss the diagnostic and predictive values of cBIN1 in future clinical use.
\end{abstract}

heart failure, cBIN1, t-tubules, calcium transient, arrhythmias

Citation: Zhou, K., and Hong, T. (2017). Cardiac BIN1 (cBIN1) is a regulator of cardiac contractile function and an emerging biomarker of heart muscle health. Sci China Life Sci 60, 257-263. doi: 10.1007/s11427-016-0249-x

\section{INTRODUCTION}

Heart failure (HF), a common end-stage syndrome of different cardiac diseases, affects millions of people worldwide (Bui et al., 2011). Although there are significant advances in its prevention, diagnosis, and treatment, effective early diagnosis and therapy of HF are still largely limited and its five-year morbidity and mortality remain high. Development of new diagnostic and therapeutic tools requires further understanding of molecular mechanisms underlying

*Corresponding author (email: TingTing.Hong@eshs.org)
HF progression. Normal cardiac contraction is initiated by a sequence of calcium signaling events requiring specific communication between L-type calcium channels (LTCC) at the transverse-tubule (t-tubule) membrane and ryanodine receptors (RyR) at the junctional sarcoplasmic reticulum (jSR) membrane. At each heartbeat, initial action potential-induced calcium entry through activated LTCCs triggers the subsequent massive calcium release from the SR store via the calcium sensing and releasing RyRs, a process known as calcium-induced-calcium-release (CICR). The synchrony of CICR determines the strength of calcium transients and the efficiency of excitation-contraction coupling (E-C coupling). 
A weakened calcium transient with E-C uncoupling is the key pathophysiology of HF. Many membrane scaffolding proteins including junctophilin 2 (JPH2), caveoline-3, and the Bin/Amphiphysin/Rvs (BAR) domain-containing protein bridging integrator 1 (BIN1, also known as amphiphysin 2) (Ibrahim et al., 2011) have been described playing essential roles in regulating E-C coupling. Of these, the cardiac isoform of BIN1, cBIN1 or BIN1+13+17, has been implicated in the formation of t-tubule membrane microfolds to cluster LTCCs and recruit phosphorylated RyRs for coupling with LTCCs. As a result, functional LTCC-RyR couplons form at these cBIN1-microdomains, controlling CICR and E-C coupling. Furthermore, BIN1 is transcriptionally reduced in failing hearts (Hong et al., 2012; Lyon et al., 2012), diminishing calcium transients and impairing cardiac contractility. Here we will review the most recent understanding of how BIN1 is coordinated to regulate the cardiac dyad system and its potential usage for HF prediction and therapy development.

\section{BIN1 AND ITS DIFFERENT ISOFORMS}

Four BAR protein-encoding genes have been identified in the mammalian system, namely, Amphiphysin I, Bin1 (also known as AmpII), Bin2, and Bin3 (Lichte et al., 1992; Sakamuro et al., 1996). All BAR genes encode protein products with a unique signature domain termed the BAR domain (Sakamuro et al., 1996). As a member of the BAR-domain containing protein superfamily, BIN1 contains a signature N-terminal BAR-domain (N-BAR) encoded by exons 1-9. The crystal structure of the N-BAR domain revealed a dimeric interaction between helixes from each monomer to form a 6-helix bundle in BIN1 dimers (Casal et al., 2006). The structure of this dimer is similar to an elongated banana shape (Peter et al., 2004; Fu and Hong, 2015). Thus, based on the BAR domain shape of BIN1 and its general function as a membrane curvature protein, BIN1 has been referred to as the "banana" molecule (Qualmann et al., 2011). BIN1 has been implicated in many cellular processes, such as inducing membrane invagination (Frost et al., 2009) and initiating tubulogenesis in skeletal muscle cells (Lee et al., 2002). In human and mouse heart cells, BIN1 localizes to cardiac t-tubules (Hong et al., 2010), forms t-tubule membrane microdomains (Hong et al., 2014; Fu et al., 2016), facilitates cytoskeleton-based calcium channel trafficking to t-tubule membrane (Hong et al., 2010), and causes RyR movement along the SR membrane (Fu et al., 2016).

Interestingly, these different cellular functions of BIN1 heavily rely on various BIN1 protein isoforms generated from tissue specific alternative splicing of a single Bin1 gene with 20 exons (Figure 1). Over ten BIN1 protein isoforms are encoded with tissue and disease specificity. Using PCR detection of the cDNA fragments containing the alternatively spliced region between exons 10 and 18 in Bin1, we cloned four protein-encoding transcripts of Bin1 mRNA in adult mouse cardiomyocytes (Hong et al., 2014): a small constitutive Bin1 (excluding exons 7, 11, and 13-17), a constitutively alternatively spliced $\operatorname{Bin} 1+17$ (Bin $1+$ exon 17 ), and two cardiac alternatively spliced variants $B i n 1+13$ and $B i n 1+13+17(c B I N 1)$. A group of four exons 13-16 is normally co-spliced together in neuronal cells to encode a clathrin-binding domain, which targets neuronal BIN1 to clathrin-coated pits to facilitate endocytosis (Taylor et al., 2011) and neurotransmitter reuptake (Butler et al., 1997). The skeletal spliced exon 11 encodes a phosphoinositide binding domain that targets skeletal BIN1 to the plasma membrane for biogenesis of skeletal t-tubules (Lee et al., 2002). Although skeletal t-tubules have marked differences from cardiac t-tubules in number, size and intracellular location, with expression of the skeletal isoform BIN $1+11+17$,

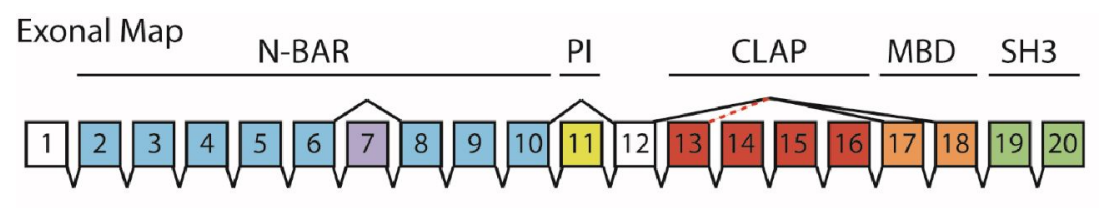

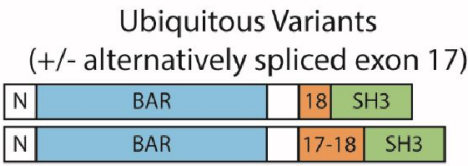

Neuornal Variants (with co-spliced exons 13 to 16)
Cardiac Variants

(with cardiac spliced exon 13)

\begin{tabular}{|l|l|l|l|l|l|}
\hline $\mathrm{N}$ & BAR & & 13 & 18 & $\mathrm{SH} 3$ \\
\hline \hline $\mathrm{N}$ & BAR & & 13 & $17-18$ & $\mathrm{SH} 3$ \\
\hline
\end{tabular}

Skeletal Variants

(with skeletal spliced exon 11)

\begin{tabular}{|l|l|l|l|l|l|}
\hline $\mathrm{N}$ & BAR & & 11 & 18 & $\mathrm{SH} 3$ \\
\hline $\mathrm{N}$ & BAR & & 11 & $17-18$ & $\mathrm{SH} 3$ \\
\hline
\end{tabular}

Figure 1 (Color online) Alternative spliced Binl transcript variants in adult mouse tissues. N-BAR, N-Bin-amphiphysin-Rvs domain; PI, phosphoinositide binding domain; CLAP, clathrin/AP2 binding region; MBD, myc binding domain; SH3, SH3 domain. 
analogous membrane microfolds may also exist within skeletal t-tubules. The most abundant BIN1 isoform in mouse hearts is BIN1+13, which is known to be associated with cell proliferation (Ge et al., 1999). On the other hand, cBIN1 $(\mathrm{BIN} 1+13+17)$, the t-tubule localized cardiac isoform, promotes N-WASP dependent actin polymerization and induces actin-dependent densely packed t-tubule membrane microfolds (Hong et al., 2014). This particular cardiac isoform cBIN1 is the only BIN1 isoform that is capable of rescuing t-tubule microfolds in transgenic mice with conditional Bin1 knockout in cardiomyocytes (Hong et al., 2014). Furthermore, cBIN1 is the isoform organizing t-tubule LTCC-RyR dyads (Fu et al., 2016). Detailed functions of other BIN1 isoforms in the heart await further studies.

\section{THE ROLE OF BIN1 IN T-TUBULE MICRODOMAIN ORGANIZATION AND FUNCTION}

In mammalian ventricular myocytes, t-tubules are well developed interconnected tubular membrane network with its primary transverse section regularly spaced in coincident with the z-lines. The t-tubule system plays a significant role in safeguarding the instantaneous excitation and synchronous activation of nearly all calcium releasing channel RyRs at the jSR membrane (Guo et al., 2013). It is well known that LTCCs collect at t-tubule membrane to be in close physical proximity to RyRs at the jSR membrane, forming LTCC-RyR dyads that facilitate CICR (Bers, 2002). Proper organization of the two dyadic proteins LTCC and RyR at the membrane microdomains is critical in maintaining normal cardiac calcium signaling and heart contractility. In heart failure, disorganization in dyads impairs CICR and diminishes calcium transients, limiting cardiac contractility.

BIN1 regulates dyad microdomain organization and function from four separate aspects (Figure 2, top panel). First, BIN1 facilitates LTCC localization to t-tubule membrane. LTCC forward trafficking to t-tubules follows "Targeted Delivery" model of channel delivery, which was first introduced for connexin 43 trafficking to cardiac intercalated discs (Shaw et al., 2007). In "Targeted Delivery", channel delivery to a specific membrane subdomain requires the dynamic microtubule highway and its coordination with channel itself and a particular membrane anchor protein. In the case of LTCC trafficking, LTCC-carrying microtubules are attracted to BIN1 at t-tubule membrane (Hong et al., 2010). Once tethered to BIN1, microtubules unload their cargo calcium channels to BIN1 protein at the membrane. Thus, serving as the membrane anchor protein for LTCCs, BIN1 causes t-tubule concentration of the channel. Second, BIN1 also facilitates the clustering of calcium channels that are already at the t-tubule membrane. Super-resolution (10-20 nm) fluorescent imaging revealed that LTCCs form large clusters at BIN1-membrnae (Fu et al., 2016), which become smaller in cardiomyocytes with heterozygous Bin1 deletion (Hong et al., 2014; Fu and Hong, 2015). Channel clustering at cBIN1-microdomains may also affect coupled-gating of LTCCs therefore normal channel function. Third, in addition to trafficking and clustering LTCCs at the t-tubule membrane, cBIN1 plays a critical role in recruiting the other dyadic protein RyRs to the jSR membrane (Fu et al., 2016). As a result, cBIN1 serves as a protein bridge important for the formation and maintenance of LTCC-RyR couplons, which are essential for normal E-C coupling. Recruitment of RyRs to cBIN1 is phosphorylation dependent (Fu et al., 2016). By recruiting S2808-phosphorylated RyRs with increased calcium sensitivity and open probability (Marx et al., 2000) to couple with LTCCs, cBIN1 increases CICR gain necessary during an acute stress response in normal hearts. Lastly, cBIN1 organizes the ultrastructure of t-tubule membrane (Hong et al., 2014) supporting these channels. This recently identified cardiac t-tubule membrane isoform cBIN1 sculpts tiny microfolds at t-tubule membrane, which not only house LTCC clusters but also create a "fuzzy space" like extracellular ionic diffusion barrier (Hong et al., 2014). With restricted diffusion of extracellular calcium ions within the local microenvironment, cBIN1 controls the driving force of LTCC currents. This protective ionic diffusion barrier becomes even more significant during fast pacing states where restricted diffusion can effectively limit a quick depletion of extracellular calcium ions, preventing loss of transmembrane ionic flux. When cBIN1 is reduced as occurs in HF (Hong et al., 2012; Lyon et al., 2012; Caldwell et al., 2014), not only channel localization to dyads is impaired, but also removal of the diffusion barrier can deplete extracellular ions, further diminishing LTCC current and impairing CICR (Figure 2, bottom panel).

Whether functions of cBIN1-microdomains involve other t-tubule scaffolding proteins including caveolin-3 and JPH2 remains unclear. Caveonlin-3 organized caveolae distributes along t-tubules and caveolae distribution is altered in Bin1deleted mouse cardiomyocytes (Laury-Kleintop et al., 2015), indicating crosstalk between cBIN1-microdomains and caveolae microdomains within t-tubules. JPH2 is a t-tubule/jSR junctional protein and is involved in the maintenance of junctional membrane structure, which when knocked down increases the distance between t-tubule and jSR membrane and disrupts jSR membrane complexes (van Oort et al., 2011; Wu et al., 2012; Reynolds et al., 2013). A role of JPH2 in HF progression has been reported (see review in (Takeshima et al., 2015)). Some studies indicate that JPH2 is down-regulated in animal models of hypertrophic and dilated cardiomyopathy (Zhang et al., 2013), and conditional knockdown of JPH2 in the heart causes systolic heart failure in murine models (Chen et al., 2013). Other studies report no significant changes of JPH2 expression in failing hearts (Caldwell et al., 2014), or 


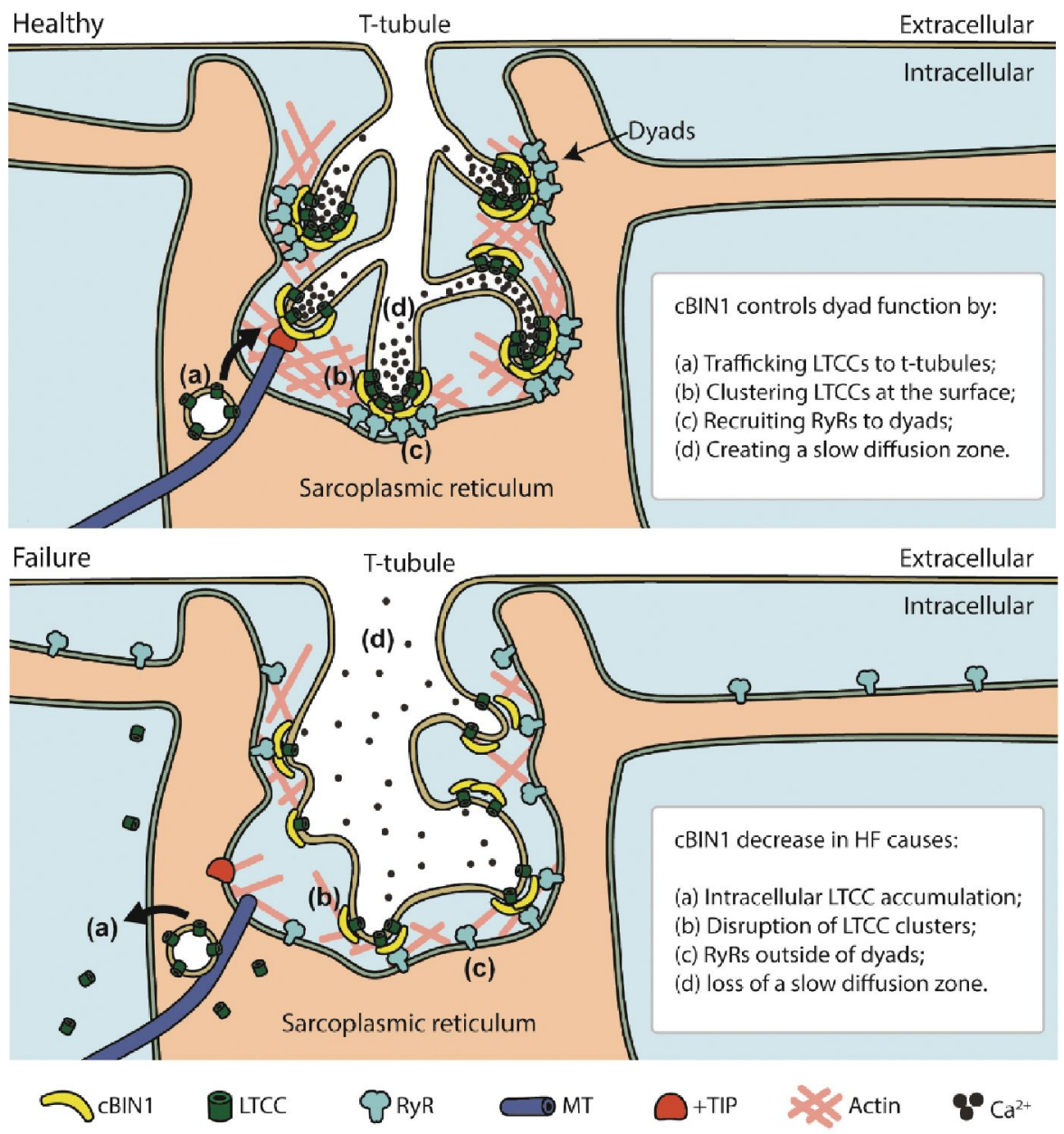

Figure 2 (Color online) Cartoon of cBIN1-microdomain regulated cardiac dyad function in healthy (top) and failing cardiomyocytes (bottom).

with recovery of heart function (Lyon et al., 2012). Whether JPH2 can facilitate cBIN1-induced LTCC-RyR dyad formation is an interesting topic. Future studies will be necessary to identify how these membrane microdomains interact with each other at t-tubules to maintain a coordinated calcium signaling event in healthy hearts and how normal interactions are disrupted in HF.

It is also worth emphasizing that the dyad-organizing cBIN1-microdomain is extremely dynamic, which reorganizes within minutes of stress response (Fu et al., $2016)$. Acute $\beta$-adrenergic receptor ( $\beta$-AR) activation redistributes cBIN1 to t-tubules, likely through accumulation of BIN1-binding phospholipids (Lee et al., 2002), which are known regulators of channel function (Fu et al., 2015). As a result of cBIN1 redistribution, phosphorylated RyRs is recruited to cBIN1-microdomains to couple with LTCCs at the t-tubule membrane. Thus, cBIN1 controls $\beta$-AR-dependent LTCC-RyR coupling at dyads during acute stress (Fu et al., 2016), improving CICR gain and calcium transient as observed previously (Zhou et al., 2009). When BIN1 is reduced, RyR recruitment into dyads will be impaired, accumulation of uncoupled receptors outside of dyads not only limits efficient CICR but also forms leaky receptor clusters, impairing cardiac contractility (Hong et al., 2012) and increasing arrhythmia risks (Hong et al., 2014). The dynamic feature of cBIN1-microdomains suggests a possible membrane turnover from the microdomains, releasing membrane and cBIN1 into circulation. As a result, cBIN1 is blood available and plasma cBIN1 is a potential indicator for myocardial health (Hong et al., 2012). Future study will be necessary to understand the molecular mechanisms controlling the life cycles of cBIN1-microdomain, its releasing mechanisms, and the biomarker potential of plasma cBIN1. On the other hand, a similar skeletal BIN1-organized membrane microdomain may also exist in skeletal t-tubules to regulate LTCC and RyR function and contribute to skeletal calcium signaling, which is important for skeletal muscle health and disease (Zhou et al., 2016).

\section{BIN1 IN HEART FAILURE PROGRESSION}

As the fastest growing cardiovascular disorder affecting over 40 million patients worldwide (Rosamond et al., 2007; LloydJones et al., 2010; Roger et al., 2011), HF still awaits further studies to understand its mechanistic progression. Such 
knowledge is in great need for effective development of new diagnostic, prognostic, and therapeutic tools for $\mathrm{HF}$, an endstage cardiac disease with poor clinical prognosis. The pathophysiologic hallmark of HF is a weakened cardiac calcium transient (Gómez et al., 1997; Litwin et al., 2000; Gómez et al., 2001), which can be attributed to t-tubule remodeling (Lyon et al., 2009; Louch et al., 2010; Wei et al., 2010) and dyad uncoupling (Gómez et al., 1997; Gómez et al., 2001; He et al., 2001; Louch et al., 2006; Bito et al., 2008; Louch et al., 2010). T-tubule remodeling marks the transition from hypertrophy to failure (Wei et al., 2010), contributing to the progression of HF (see reviews in (Brette and Orchard, 2003; Brette and Orchard, 2007; Louch et al., 2010; Ferrantini et al., 2013; Guo et al., 2013; Ibrahim and Terracciano, 2013)). Molecules like cBIN1 with the ability to modulate t-tubule and dyad function are promising targets for future therapy development. Given its roles in organizing t-tubule microfolds and dyad microdomains, cBIN1 has emerged as a crucial regulator of cardiac function in healthy and diseased hearts (Hong et al., 2012; Laury-Kleintop et al., 2015).

The role of BIN1 in mechanistic HF progression was first noted when constitutive Bin1 knockout mice died of perinatal lethal cardiomyopathy (Muller et al., 2003). Later, a decrease in BIN1 transcription was identified in acquired human HF (Hong et al., 2012) and multiple animal models of HF (Lyon et al., 2012; Caldwell et al., 2014), which recovers during functional recovery (Lyon et al., 2012). In failing cardiomyocytes, decreased BIN1 level impairs LTCC trafficking (Hong et al., 2012; Lyon et al., 2012; Caldwell et al., 2014), diminishing calcium transients. In zebrafish, knockdown of Binl induces significant contractile dysfunction and cardiomyopathy (Hong et al., 2012). Mice with cardiac conditional knockout of Bin1 gene developed dilated cardiomyopathy when aged (starting at 8-10 months of age) or with pressure overload (Laury-Kleintop et al., 2015). Before decompensation into contractile dysfunction, young adult mice (2-4 months of age) with Bin1 deletion already have increased risks of ventricular arrhythmias. These data indicate that in HF with reduced cBIN1 expression, disruption in cBIN1-microdomains not only impairs the dyadic calcium releasing units, but also removes microfold-created protective slow diffusion zone (Figure 2, bottom panel), increasing susceptibility to ventricular arrhythmias (Hong et al., 2014). Taken together, compromised cBIN1 contributes a pivotal role in inducing both contractile pump failure and electrical instability of failing hearts. Thus, cBIN1 based microdomain is a potential therapeutic target for new HF treatment. Future studies are necessary to identify whether reduced Binl transcription or aberrant splicing of cardiac exon 13 is responsible for cardiomyopathy development.

On the other hand, cBIN1 is a potential biomarker of $\mathrm{HF}$, which directly measures the biochemical health of cardiomyocytes. Myocardial cBIN1 is released into circulation possibly through a continuous turnover process of cBIN1-microdomain, providing a blood index of myocardial health (Hong et al., 2012). In a cohort of arrhythmogenic right ventricular cardiomyopathy patients, plasma BIN1 decreases in patients with HF symptoms (Hong et al., 2012). Low plasma levels of BIN1 correlates with HF status, and predicts future arrhythmia incidences (Hong et al., 2012). A cardiac specific blood test detecting the recently cloned functional significant cBIN1 (Hong et al., 2014) is currently under development, which when succeeded will greatly aid in the diagnosis, prognosis and therapy guidance of HF patients.

\section{CONCLUSIONS}

As a multifunctional regulator of dyads at cardiac t-tubules, BIN1 controls physiological calcium signaling in healthy cardiomyocytes, and reduced BIN1 contributes to pathophysiology of HF progression. Thus, understanding BIN1 biology in normal and heart failure will have a great impact in disease management and guided therapy. The identified cBIN1-microdomain may serve as a target for therapeutic development. Understanding the mechanistic decrease of BIN1 in HF may also help identify new pathways that can be targeted for new therapy development. On the other hand, with its blood availability, plasma BIN1 carries a message of cardiac health and serves as a potential biomarker of the biochemical health and recovery potential of hearts. Developing a cardiac specific cBIN1 assay, understanding the metabolic characteristics and kinetics of BIN1 in human plasma, and learning other potential confounding factors of plasma BIN1 are all necessary steps to define a clinical test for risk stratification and therapy guidance in millions of patients with acquired heart failure.

Compliance and ethics The author(s) declare that they have no conflict of interest.

Acknowledgements This work was supported by the United States National Institute of Health/National Heart, Lung, and Blood Institute (NIH/NHLBI, Hong R01 HL133286), and American Heart Association (AHA) (IRG27780031, BGIA27770151).

Bers, D.M. (2002). Cardiac Excitation-Contraction Coupling. (New York: Springer), pp. 198-205.

Bito, V., Heinzel, F.R., Biesmans, L., Antoons, G., and Sipido, K.R. (2008). Crosstalk between L-type $\mathrm{Ca}^{2+}$ channels and the sarcoplasmic reticulum: alterations during cardiac remodelling. Cardiovasc Res 77, 315-324.

Brette, F., and Orchard, C. (2003). T-tubule function in mammalian cardiac myocytes. Circul Res 92, 1182-1192.

Brette, F., and Orchard, C. (2007). Resurgence of cardiac t-tubule research. Physiology 22, 167-173.

Bui, A.L., Horwich, T.B., and Fonarow, G.C. (2011). Epidemiology and risk profile of heart failure. Nat Rev Cardiol 8, 30-41.

Butler, M.H., David, C., Ochoa, G.C., Freyberg, Z., Daniell, L., Grabs, D., Cremona, O., and Camilli, P.D. (1997). Amphiphysin II (SH3P9; BIN1), a member of the amphiphysin/Rvs family, is concentrated in the cortical cytomatrix of axon initial segments and nodes of ranvier in brain and 
around T tubules in skeletal muscle. J Cell Biol 137, 1355-1367.

Caldwell, J.L., Smith, C.E.R., Taylor, R.F., Kitmitto, A., Eisner, D.A., Dibb, K.M., and Trafford, A.W. (2014). Dependence of cardiac transverse tubules on the BAR domain protein amphiphysin II (BIN-1). Circ Res 115, 986-996.

Casal, E., Federici, L., Zhang, W., Fernandez-Recio, J., Priego, E.M., Miguel, R.N., DuHadaway, J.B., Prendergast, G.C., Luisi, B.F., and Laue, E.D. (2006). The crystal structure of the BAR domain from human Bin1/amphiphysin II and its implications for molecular recognition. Biochem 45, 12917-12928.

Chen, B., Guo, A., Zhang, C., Chen, R., Zhu, Y., Hong, J., Kutschke, W., Zimmerman, K., Weiss, R.M., Zingman, L., Anderson, M.E., Wehrens, X.H.T., and Song, L.S. (2013). Critical roles of junctophilin-2 in T-tubule and excitation-contraction coupling maturation during postnatal development. Cardiovasc Res 100, 54-62.

Ferrantini, C., Crocini, C., Coppini, R., Vanzi, F., Tesi, C., Cerbai, E., Poggesi, C., Pavone, F.S., and Sacconi, L. (2013). The transverse-axial tubular system of cardiomyocytes. Cell Mol Life Sci 70, 4695-4710.

Frost, A., Unger, V.M., and De Camilli, P. (2009). The BAR domain superfamily: membrane-molding macromolecules. Cell 137, 191-196.

Fu, J., Gao, Z.B., Shen, B., and Zhu, M.X. (2015). Canonical transient receptor potential 4 and its small molecule modulators. Sci China Life Sci 58, 39-47.

Fu, Y., and Hong, T. (2015). BIN1 regulates dynamic t-tubule membrane. Biochim Biophys Acta 1863, 1839-1847.

Fu, Y., Shaw, S.A., Naami, R., Vuong, C.L., Basheer, W.A., Guo, X., and Hong, T.T. (2016). Isoproterenol promotes rapid ryanodine receptor movement to bridging integrator 1 (BIN1)-organized dyads. Circulation 133, 388-397.

Ge, K., DuHadaway, J., Du, W., Herlyn, M., Rodeck, U., and Prendergast, G.C. (1999). Mechanism for elimination of a tumor suppressor: aberrant splicing of a brain-specific exon causes loss of function of Bin1 in melanoma. Proc Natl Acad Sci USA 96, 9689-9694.

Gómez, A.M., Guatimosim, S., Dilly, K.W., Vassort, G., and Lederer, W.J. (2001). Heart failure after myocardial infarction: altered excitation-contraction coupling. Circulation 104, 688-693.

Gómez, A.M., Valdivia, H.H., Cheng, H., Lederer, M.R., Santana, L.F., Cannell, M.B., McCune, S.A., Altschuld, R.A., and Lederer, W.J. (1997). Defective excitation-contraction coupling in experimental cardiac hypertrophy and heart failure. Science 276, 800-806.

Guo, A., Zhang, C., Wei, S., Chen, B., and Song, L.S. (2013). Emerging mechanisms of T-tubule remodelling in heart failure. Cardiovasc Res 98, 204-215.

He, J., Conklin, M.W., Foell, J.D., Wolff, M.R., Haworth, R.A., Coronado, R., and Kamp, T.J. (2001). Reduction in density of transverse tubules and L-type $\mathrm{Ca}^{2+}$ channels in canine tachycardia-induced heart failure. Cardiovasc Res 49, 298-307.

Hong, T.T., Yang, H., Zhang, S.S., Cho, H.C., Kalashnikova, M., Sun, B., Zhang, H., Bhargava, A., Grabe, M., Olgin, J., Gorelik, J., Marbán, E., Jan, L.Y., and Shaw, R.M. (2014). Cardiac BIN1 folds T-tubule membrane, controlling ion flux and limiting arrhythmia. Nat Med 20, 624-632.

Hong, T.T., Cogswell, R., James, C.A., Kang, G., Pullinger, C.R., Malloy, M.J., Kane, J.P., Wojciak, J., Calkins, H., Scheinman, M.M., Tseng, Z.H., Ganz, P., De Marco, T., Judge, D.P., and Shaw, R.M. (2012). Plasma BIN1 correlates with heart failure and predicts arrhythmia in patients with arrhythmogenic right ventricular cardiomyopathy. Heart Rhythm 9, 961-967.

Hong, T.T., Smyth, J.W., Chu, K.Y., Vogan, J.M., Fong, T.S., Jensen, B.C., Fang, K., Halushka, M.K., Russell, S.D., Colecraft, H., Hoopes, C.W., Ocorr, K., Chi, N.C., and Shaw, R.M. (2012). BIN1 is reduced and Cav1.2 trafficking is impaired in human failing cardiomyocytes. Heart Rhythm 9, 812-820.

Hong, T.T., Smyth, J.W., Gao, D., Chu, K.Y., Vogan, J.M., Fong, T.S., Jensen, B.C., Colecraft, H.M., and Shaw, R.M. (2010). BIN1 localizes the L-type calcium channel to cardiac T-tubules. PLoS Biol 8, e1000312.
Ibrahim, M., Gorelik, J., Yacoub, M.H., and Terracciano, C.M. (2011). The structure and function of cardiac t-tubules in health and disease. Proc R Soc B-Biol Sci 278, 2714-2723.

Ibrahim, M., and Terracciano, C.M. (2013). Reversibility of T-tubule remodelling in heart failure: mechanical load as a dynamic regulator of the T-tubules. Cardiovasc Res 98, 225-232.

Laury-Kleintop, L.D., Mulgrew, J.R., Heletz, I., Nedelcoviciu, R.A., Chang, M.Y., Harris, D.M., Koch, W.J., Schneider, M.D., Muller, A.J., and Prendergast, G.C. (2015). Cardiac-specific disruption of Bin1 in mice enables a model of stress- and age-associated dilated cardiomyopathy. J Cell Biochem 116, 2541-2551.

Lee, E., Marcucci, M., Daniell, L., Pypaert, M., Weisz, O.A., Ochoa, G.C., Farsad, K., Wenk, M.R., and De Camilli, P. (2002). Amphiphysin 2 (Bin1) and T-tubule biogenesis in muscle. Science 297, 1193-1196.

Lichte, B., Veh, R. W., Meyer, H. E., and Kilimann, M. W. (1992). Amphiphysin, a novel protein associated with synaptic vesicles. EMBO J 11, 2521-2530.

Litwin, S.E., Zhang, D., and Bridge, J.H.B. (2000). Dyssynchronous Ca ${ }^{2+}$ sparks in myocytes from infarcted hearts. Circ Res 87, 1040-1047.

Lloyd-Jones, D., Adams, R.J., Brown, T.M., Carnethon, M., Dai, S., De Simone, G., Ferguson, T.B., Ford, E., Furie, K., Gillespie, C., Go, A., Greenlund, K., Haase, N., Hailpern, S., Ho, P.M., Howard, V., Kissela, B., Kittner, S., Lackland, D., Lisabeth, L., Marelli, A., McDermott, M.M., Meigs, J., Mozaffarian, D., Mussolino, M., Nichol, G., Roger, V.L., Rosamond, W., Sacco, R., Sorlie, P., Stafford, R., Thom, T., Wasserthiel-Smoller, S., Wong, N.D., and Wylie-Rosett, J. (2010). Heart disease and stroke statistics - 2010 update: a report from the american heart association. Circulation 121, e46-e215.

Louch, W.E., Mørk, H.K., Sexton, J., Strømme, T.A., Laake, P., Sjaastad, I., and Sejersted, O.M. (2006). T-tubule disorganization and reduced synchrony of $\mathrm{Ca}^{2+}$ release in murine cardiomyocytes following myocardial infarction. J Physiol 574, 519-533.

Louch, W.E., Sejersted, O.M., and Swift, F. (2010). There goes the neighborhood: pathological alterations in t-tubule morphology and consequences for cardiomyocyte $\mathrm{Ca}^{2+}$ handling. J Biomed Biotech 2010, 1-17.

Lyon, A.R., MacLeod, K.T., Zhang, Y., Garcia, E., Kikonda Kanda, G., Lab, M.J., Korchev, Y.E., Harding, S.E., and Gorelik, J. (2009). Loss of T-tubules and other changes to surface topography in ventricular myocytes from failing human and rat heart. Proc Natl Acad Sci USA 106, 6854-6859.

Lyon, A.R., Nikolaev, V.O., Miragoli, M., Sikkel, M.B., Paur, H., Benard, L., Hulot, J.S., Kohlbrenner, E., Hajjar, R.J., Peters, N.S., Korchev, Y.E., Macleod, K.T., Harding, S.E., and Gorelik, J. (2012). Plasticity of surface structures and $\beta_{2}$-adrenergic receptor localization in failing ventricular cardiomyocytes during recovery from heart failure. Circ Heart Fail 5, 357-365.

Marx, S.O., Reiken, S., Hisamatsu, Y., Jayaraman, T., Burkhoff, D., Rosemblit, N., and Marks, A.R. (2000). PKA phosphorylation dissociates FKBP12.6 from the calcium release channel (ryanodine receptor). Cell 101, 365-376.

Muller, A.J., Baker, J.F., DuHadaway, J.B., Ge, K., Farmer, G., Donover, P.S., Meade, R., Reid, C., Grzanna, R., Roach, A.H., Shah, N., Soler, A.P., and Prendergast, G.C. (2003). Targeted disruption of the murine Bin1/amphiphysin ii gene does not disable endocytosis but results in embryonic cardiomyopathy with aberrant myofibril formation. Mol Cell Biol 23, 4295-4306.

Peter, B.J., Kent, H.M., Mills, I.G., Vallis, Y., Butler, P.J.G., Evans, P.R., and McMahon, H.T. (2004). BAR domains as sensors of membrane curvature: the amphiphysin BAR structure. Science 303, 495-499.

Qualmann, B., Koch, D., and Kessels, M.M. (2011). Let's go bananas: revisiting the endocytic BAR code. EMBO J 30, 3501-3515.

Reynolds, J.O., Chiang, D.Y., Wang, W., Beavers, D.L., Dixit, S.S., Skapura, D.G., Landstrom, A.P., Song, L.S., Ackerman, M.J., and Wehrens, X.H.T. (2013). Junctophilin-2 is necessary for T-tubule maturation during mouse heart development. Cardiovasc Res 100, 44-53.

Roger, V.L., Go, A.S., Lloyd-Jones, D.M., Adams, R.J., Berry, J.D., Brown, 
T.M., Carnethon, M.R., Dai, S., de Simone, G., Ford, E.S., Fox, C.S., Fullerton, H.J., Gillespie, C., Greenlund, K.J., Hailpern, S.M., Heit, J.A., Ho, P.M., Howard, V.J., Kissela, B.M., Kittner, S.J., Lackland, D.T., Lichtman, J.H., Lisabeth, L.D., Makuc, D.M., Marcus, G.M., Marelli, A., Matchar, D.B., McDermott, M.M., Meigs, J.B., Moy, C.S., Mozaffarian, D., Mussolino, M.E., Nichol, G., Paynter, N.P., Rosamond, W.D., Sorlie, P.D., Stafford, R.S., Turan, T.N., Turner, M.B., Wong, N.D., Wylie-Rosett, J., Roger, V.L., and Turner, M.B. (2011). Heart disease and stroke statistics - 2011 update: a report from the american heart association. Circulation 123, e18-e209.

Rosamond, W., Flegal, K., Friday, G., Furie, K., Go, A., Greenlund, K., Haase, N., Ho, M., Howard, V., Kissela, B., Kissela, B., Kittner, S., Lloyd-Jones, D., McDermott, M., Meigs, J., Moy, C., Nichol, G., O'Donnell, C.J., Roger, V., Rumsfeld, J., Sorlie, P., Steinberger, J., Thom, T., Wasserthiel-Smoller, S., Hong, Y., and Hong, Y. (2007). Heart disease and stroke statistics -2007 update: a report from the american heart association statistics committee and stroke statistics subcommittee. Circulation 115, e69-e171.

Sakamuro, D., Elliott, K.J., Wechsler-Reya, R., and Prendergast, G.C. (1996). BIN1 is a novel MYC-interacting protein with features of a tumour suppressor. Nat Genet 14, 69-77.

Shaw, R.M., Fay, A.J., Puthenveedu, M.A., von Zastrow, M., Jan, Y.N., and Jan, L.Y. (2007). Microtubule plus-end-tracking proteins target gap junctions directly from the cell interior to adherens junctions. Cell 128, $547-560$.

Takeshima, H., Hoshijima, M., and Song, L.S. (2015). $\mathrm{Ca}^{2+}$ microdomains organized by junctophilins. Cell Calcium 58, 349-356.
Taylor, M.J., Perrais, D., and Merrifield, C.J. (2011). A high precision survey of the molecular dynamics of mammalian clathrin-mediated endocytosis. PLoS Biol 9, e1000604.

van Oort, R.J., Garbino, A., Wang, W., Dixit, S.S., Landstrom, A.P., Gaur, N., De Almeida, A.C., Skapura, D.G., Rudy, Y., Burns, A.R., Ackerman, M.J., and Wehrens, X.H.T. (2011). Disrupted junctional membrane complexes and hyperactive ryanodine receptors after acute junctophilin knockdown in mice. Circulation 123, 979-988.

Wei, S., Guo, A., Chen, B., Kutschke, W., Xie, Y.P., Zimmerman, K., Weiss, R.M., Anderson, M.E., Cheng, H., and Song, L.S. (2010). T-tubule remodeling during transition from hypertrophy to heart failure. Circul Res $107,520-531$.

Wu, H.D., Xu, M., Li, R.C., Guo, L., Lai, Y.S., Xu, S.M., Li, S.F., Lü, Q.L., Li, L.L., Zhang, H.B., Zhang, Y.Y., Zhang, C.M., and Wang, S.Q. (2012). Ultrastructural remodelling of $\mathrm{Ca}^{2+}$ signalling apparatus in failing heart cells. Cardiovasc Res 95, 430-438.

Zhang, H.B., Li, R.C., Xu, M., Xu, S.M., Lai, Y.S., Wu, H.D., Xie, X.J., Gao, W., Ye, H., Zhang, Y.Y., Meng, X., and Wang, S.Q. (2013). Ultrastructural uncoupling between T-tubules and sarcoplasmic reticulum in human heart failure. Cardiovasc Res 98, 269-276.

Zhou, J., Dhakal, K., and Yi, J. (2016). Mitochondrial $\mathrm{Ca}^{2+}$ uptake in skeletal muscle health and disease. Sci China Life Sci 59, 770-776.

Zhou, P., Zhao, Y.T., Guo, Y.B., Xu, S.M., Bai, S.H., Lakatta, E.G., Cheng, H., Hao, X.M., and Wang, S.Q. (2009). $\beta$-Adrenergic signaling accelerates and synchronizes cardiac ryanodine receptor response to a single L-type $\mathrm{Ca}^{2+}$ channel. Proc Natl Acad Sci USA 106, 18028-18033.

Open Access This article is distributed under the terms of the Creative Commons Attribution License which permits any use, distribution, and reproduction in any medium, provided the original author(s) and source are credited. 